\title{
Antiepileptic drugs have a dose-dependent association with malformations in offspring of women with epilepsy
}

The risk of malformations in offspring is increased in a dose-dependent manner by monotherapy with any of the four major antiepileptic drugs during pregnancy. This association was identified by the EURAP Study Group, in a large observational study using data from physicians in many countries.

Women with epilepsy normally continue treatment during pregnancy, as seizures can be harmful to both the mother and the developing fetus. Research has shown, however, that many antiepileptic drugs are teratogenic, increasing the risk of malformations in the offspring. Tomson explained that he and his colleagues aimed to use data from the EURAP epilepsy and pregnancy registry "to compare different treatment with regard to risks for major congenital malformations."

The EURAP registry includes observational data from physicians in 42 countries. Prospective data were recorded from pregnant women at conception-providing information about antiepileptic treatment and other risk factors-and pregnancy outcome was later recorded. 3,909 pregnancies were included in the analysis, from mothers being treated with carbamazepine, lamotrigine, valproic acid or phenobarbitol.

Treatment during pregnancy with any of these four drugs was associated with an increased chance of malformation in the offspring, and these associations were all dose-dependent. Particularly high malformation rates were recorded for doses of 1,500 mg per day or higher of valproic acid, and the lowest rates were found for treatment with less than $300 \mathrm{mg}$ per day of lamotrigine.

By examining a large cohort, the researchers were able to compare the relative teratogenicity of drugs used in antiepileptic treatment, and to remove many covariates from the analysis. "These results ... provide the prescriber with the possibility to compare the risks with a particular drug at a specific dose with
44 ... better understanding ... will help to make treatment both as safe and as effective as possible 77

risks with other treatment alternatives," says Tomson.

The researchers hope that better understanding of the relative risks of epilepsy treatment will help to make treatment both as safe and as effective as possible. They plan to "collect more pregnancies to assess less frequently used antiepileptic drugs, to look at risks with combinations of different drugs and to assess the risks for individual, specific congenital malformations."

Eleanor Beal

Original article Tomson, T. et al. Dose-dependent risk of malformations with antiepileptic drugs: an analysis of data from the EURAP epilepsy and pregnancy registry. Lancet Neurol. 10, 609-617 (2011) 